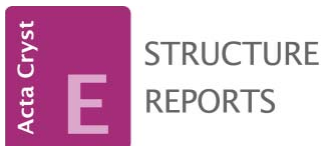

OPEN $\curvearrowright$ ACCESS

ISSN 1600-5368

\section{Crystal structure of 5-fluoro-2-(3-fluoro- phenyl)-3-methylsulfinyl-1-benzofuran}

\section{Hong Dae Choi ${ }^{\mathrm{a}}$ and Uk Lee ${ }^{\mathrm{b} *}$}

${ }^{a}$ Department of Chemistry, Dongeui University, San 24 Kaya-dong, Busanjin-gu, Busan 614-714, Republic of Korea, and ${ }^{\mathbf{b}}$ Department of Chemistry, Pukyong National University, 599-1 Daeyeon 3-dong, Nam-gu, Busan 608-737, Republic of Korea. *Correspondence e-mail: uklee@pknu.ac.kr

Received 8 October 2014; accepted 13 October 2014

Edited by L. Fabian, University of East Anglia, England

In the title compound, $\mathrm{C}_{15} \mathrm{H}_{10} \mathrm{~F}_{2} \mathrm{O}_{2} \mathrm{~S}$, the dihedral angle between the planes of the benzofuran ring system [r.m.s. deviation $=0.015(1) \AA]$ and the 3-fluorophenyl ring is $26.60(5)^{\circ}$. In the crystal, molecules are linked by $\mathrm{C}-\mathrm{H} \cdots \mathrm{O}$ and $\mathrm{C}-\mathrm{H} \cdots \mathrm{F}$ hydrogen bonds, and by $\pi-\pi$ interactions between the benzofuran rings of inversion-related molecules [centroid(benzene)-centroid(furan) distance $=3.819(2) \AA]$, forming a three-dimensional network.

Keywords: crystal structure; benzofuran; 3-fluorophenyl; $\pi-\pi$ interactions..

CCDC reference: 1029041

\section{Related literature}

For a related structure and background to benzofuran derivatives, see: Choi \& Lee (2014). For further synthetic details, see: Choi et al. (1999).

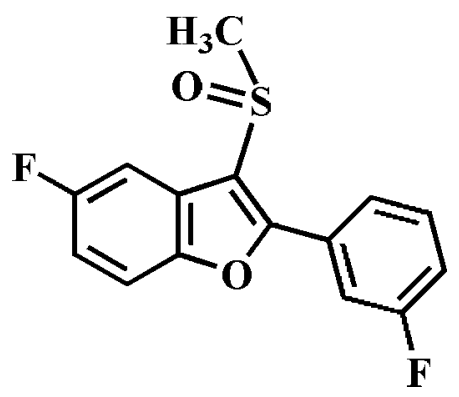

\section{Experimental}

2.1. Crystal data

$\mathrm{C}_{15} \mathrm{H}_{10} \mathrm{~F}_{2} \mathrm{O}_{2} \mathrm{~S}$

$M_{r}=292.29$

Monoclinic, $P 2_{1} / c$

$a=8.4826$ (2) A

$b=16.6307(4) \AA$

$c=9.7493(2) \AA$

$\beta=113.756(1)^{\circ}$

$V=1258.81(5) \AA^{3}$

$Z=4$

Mo $K \alpha$ radiation

$\mu=0.28 \mathrm{~mm}^{-1}$

$T=173 \mathrm{~K}$

$0.62 \times 0.55 \times 0.42 \mathrm{~mm}$

\subsection{Data collection}

Bruker SMART APEXII CCD diffractometer

Absorption correction: multi-scan (SADABS; Bruker, 2009)

$T_{\min }=0.846, T_{\max }=0.892$

12021 measured reflections

3111 independent reflections 2736 reflections with $I>2 \sigma(I)$

$R_{\text {int }}=0.027$

\subsection{Refinement}

$R\left[F^{2}>2 \sigma\left(F^{2}\right)\right]=0.036$

$w R\left(F^{2}\right)=0.098$

$S=1.03$

3111 reflections

182 parameters

$\mathrm{H}$-atom parameters constrained

$\Delta \rho_{\max }=0.41{\mathrm{e} \AA^{-3}}^{-3}$

$\Delta \rho_{\min }=-0.35$ e $\AA^{-3}$

Table 1

Hydrogen-bond geometry $\left(\AA{ }^{\circ}\right)$.

\begin{tabular}{llllll}
\hline$D-\mathrm{H} \cdots A$ & $D-\mathrm{H}$ & $\mathrm{H} \cdots A$ & $D \cdots A$ & \multicolumn{2}{l}{$D-\mathrm{H} \cdots A$} \\
\hline $\mathrm{C} 5-\mathrm{H} 5 \cdots \mathrm{O} 2^{\mathrm{i}}$ & 0.95 & 2.45 & $3.2612(18)$ & 143 & \\
$\mathrm{C} 12-\mathrm{H} 12 \cdots \mathrm{O} 2^{\mathrm{ii}}$ & 0.95 & 2.42 & $3.3361(19)$ & 161 & \\
$\mathrm{C} 15-\mathrm{H} 15 A \cdots \mathrm{F} 1^{\mathrm{iii}}$ & 0.98 & 2.54 & $3.409(2)$ & 147 & \\
$\mathrm{C} 15-\mathrm{H} 15 B \cdots \mathrm{F} 2^{\text {iv }}$ & 0.98 & 2.55 & $3.163(2)$ & 121 & \\
\hline Symmetry codes: & (i) $-x+2, y-\frac{1}{2},-z+\frac{3}{2} ;$ & (ii) $x-1, y, z-1 ;$ & (iii) \\
$-x+2,-y+1,-z+2 ;$ (iv) $x, y, z+1$. & & & & &
\end{tabular}

Data collection: APEX2 (Bruker, 2009); cell refinement: SAINT (Bruker, 2009); data reduction: SAINT; program(s) used to solve structure: SHELXS97 (Sheldrick, 2008); program(s) used to refine structure: SHELXL97 (Sheldrick, 2008); molecular graphics: ORTEP-3 for Windows (Farrugia, 2012) and DIAMOND (Brandenburg, 1998); software used to prepare material for publication: SHELXL97.

\section{Acknowledgements}

The X-ray centre of the Gyeongsang National University is acknowledged for providing access to the single-crystal diffractometer.

Supporting information for this paper is available from the IUCr electronic archives (Reference: FY2119).

\section{References}

Brandenburg, K. (1998). DIAMOND. Crystal Impact GbR, Bonn, Germany. Bruker (2009). APEX2, SADABS and SAINT. Bruker AXS Inc., Madison, Wisconsin, USA.

Choi, H. D. \& Lee, U. (2014). Acta Cryst. E70, o991-o992.

Choi, H. D., Seo, P. J. \& Son, B. W. (1999). J. Korean Chem. Soc. 43, 606-608. Farrugia, L. J. (2012). J. Appl. Cryst. 45, 849-854.

Sheldrick, G. M. (2008). Acta Cryst. A64, 112-122. 


\section{supporting information}

Acta Cryst. (2014). E70, o1168 [doi:10.1107/S160053681402251X]

\section{Crystal structure of 5-fluoro-2-(3-fluorophenyl)-3-methylsulfinyl-1-benzofuran}

\section{Hong Dae Choi and Uk Lee}

\section{S1. Comment}

As a part of our continuing program for benzofuran derivatives (Choi \& Lee, 2014), we report herein on the crystal structure of the title compound.

In the title molecule (Fig. 1), the benzofuran unit is essentially planar, with a mean deviation of 0.015 (1) $\AA$ from the least-squares plane defined by the nine constituent atoms. The 3-fluorophenyl ring is essentially planar, with a mean deviation of 0.009 (1) $\AA$ from the least-squares plane defined by the six constituent atoms. The dihedral angle formed by the benzofuran ring and the 3-fluorophenyl ring is $26.60(5)^{\circ}$. In the crystal structure (Fig. 2), molecules are linked by $\mathrm{C}$ $-\mathrm{H} \cdots \mathrm{O}$ and $\mathrm{C}-\mathrm{H} \cdots \mathrm{F}$ hydrogen bonds (Table 1), and by $\pi-\pi$ interactions between the benzene and furan rings of neighbouring molecules, with a $C g 1 \cdots C g 2^{\mathrm{v}}$ distance of 3.819 (2) $\AA$ and an interplanar distance of 3.283 (2) $\AA$ resulting in a slippage of 1.951 (2) $\AA$ ( $C g 1$ and $C g 2$ are the centroids of the $\mathrm{C} 2-\mathrm{C} 7$ benzene ring and $\mathrm{C} 1 / \mathrm{C} 2 / \mathrm{C} 7 / \mathrm{O} 1 / \mathrm{C} 8$ furan ring, respectively), forming a three-dimensional network.

\section{S2. Experimental}

The starting material 5-fluoro-2-(3-fluorophenyl)-3-methylsulfanyl-1-benzofuran was prepared by literature method (Choi et al., 1999). 3-Chloroperoxybenzoic acid (77\%, $269 \mathrm{mg}, 1.2 \mathrm{mmol})$ was added in small portions to a stirred solution of 5-fluoro-2-(3-fluorophenyl)-3-methylsulfanyl-1-benzofuran (304 mg, $1.1 \mathrm{mmol}$ ) in dichloromethane (30 ml) at $273 \mathrm{~K}$. After being stirred at room temperature for $8 \mathrm{~h}$, the mixture was washed with saturated sodium bicarbonate solution $(2 \times 10 \mathrm{ml})$ and the organic layer was separated, dried over magnesium sulfate, filtered and concentrated at reduced pressure. The residue was purified by column chromatography (hexane-ethyl acetate, 1:2 v/v) to afford the title compound as a colorless solid [yield 71\% (207 mg); m.p. 459-460 K; $R_{\mathrm{f}}=0.48$ (hexane-ethyl acetate, 1:2 v/v)]. Single crystals suitable for X-ray diffraction were prepared by slow evaporation of a solution of the title compound $(24 \mathrm{mg}) \mathrm{in}$ acetone $(20 \mathrm{ml})$ at room temperature.

\section{S3. Refinement}

All $\mathrm{H}$ atoms were positioned geometrically and refined using a riding model, with $\mathrm{C}-\mathrm{H}=0.95 \AA$ for aryl, and $0.98 \AA$ for methyl $\mathrm{H}$ atoms, respectively. $U_{\text {iso }}(\mathrm{H})=1.2 U_{\text {eq }}(\mathrm{C})$ for aryl and $1.5 U_{\text {eq }}(\mathrm{C})$ for methyl $\mathrm{H}$ atoms. The rotations of methyl groups were optimized using the SHELXL-97 command AFIX 137 (Sheldrick, 2008). 


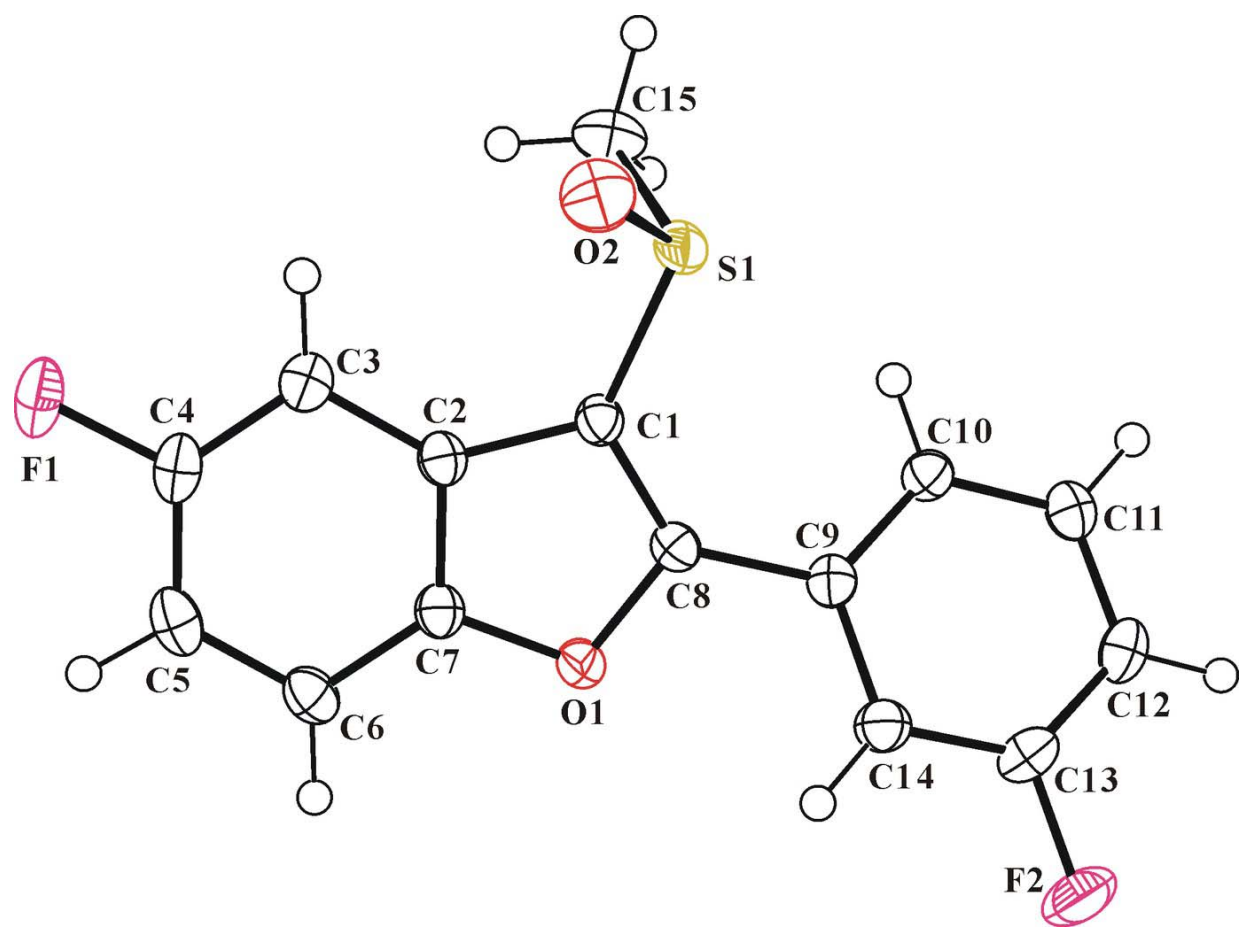

\section{Figure 1}

The molecular structure of the title compound with the atom numbering scheme. Displacement ellipsoids are drawn at the $50 \%$ probability level. $\mathrm{H}$ atoms are presented as small spheres of arbitrary radius. 


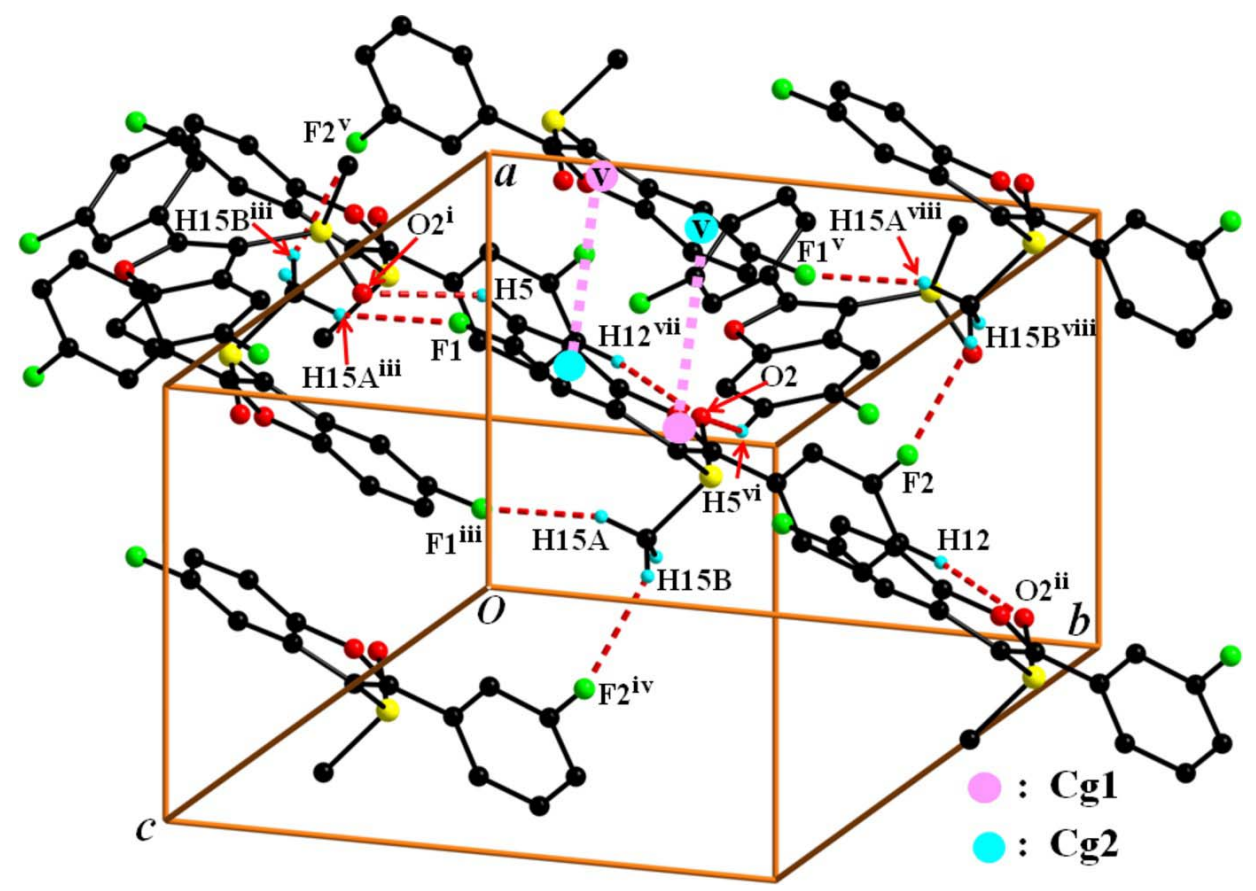

Figure 2

A view of the $\mathrm{C}-\mathrm{H} \cdots \mathrm{O}, \mathrm{C}-\mathrm{H} \cdots \mathrm{F}$ and $\pi-\pi$ interactions (dotted lines) in the crystal structure of the title compound. $\mathrm{H}$ atoms not participating in hydrogen-bonding were omitted for clarity. [Symmetry codes: (i) $-x+2, y-1 / 2,-z+3 / 2$; (ii) $x$ - 1, y,z - 1; (iii) - $x+2,-y+1,-z+2$; (iv) $x, y, z+1$; (v) $-x+2,-y+1,-z+1$; (vi) $-x+2, y+1 / 2,-z+3 / 2$; (vii) $x+1$, $y, z+1 ;($ viii) $-x+2, y+1 / 2,-z+3 / 2$.]

\section{5-Fluoro-2-(3-fluorophenyl)-3-methylsulfinyl-1-benzofuran}

Crystal data

$\mathrm{C}_{15} \mathrm{H}_{10} \mathrm{~F}_{2} \mathrm{O}_{2} \mathrm{~S}$

$M_{r}=292.29$

Monoclinic, $P 2_{1} / c$

Hall symbol: -P $2 \mathrm{ybc}$

$a=8.4826(2) \AA$

$b=16.6307(4) \AA$

$c=9.7493(2) \AA$

$\beta=113.756(1)^{\circ}$

$V=1258.81(5) \AA^{3}$

$Z=4$

\section{Data collection}

\section{Bruker SMART APEXII CCD}

diffractometer

Radiation source: rotating anode

Graphite multilayer monochromator

Detector resolution: 10.0 pixels $\mathrm{mm}^{-1}$

$\varphi$ and $\omega$ scans

Absorption correction: multi-scan

(SADABS; Bruker, 2009)

$T_{\min }=0.846, T_{\max }=0.892$
$F(000)=600$

$D_{\mathrm{x}}=1.542 \mathrm{Mg} \mathrm{m}^{-3}$

Melting point $=460-459 \mathrm{~K}$

Mo $K \alpha$ radiation, $\lambda=0.71073 \AA$

Cell parameters from 5007 reflections

$\theta=2.5-28.3^{\circ}$

$\mu=0.28 \mathrm{~mm}^{-1}$

$T=173 \mathrm{~K}$

Block, colourless

$0.62 \times 0.55 \times 0.42 \mathrm{~mm}$

12021 measured reflections

3111 independent reflections

2736 reflections with $I>2 \sigma(I)$

$R_{\text {int }}=0.027$

$\theta_{\text {max }}=28.3^{\circ}, \theta_{\text {min }}=2.5^{\circ}$

$h=-11 \rightarrow 11$

$k=-18 \rightarrow 22$

$l=-12 \rightarrow 12$ 


\section{Refinement}

Refinement on $F^{2}$

Least-squares matrix: full

$R\left[F^{2}>2 \sigma\left(F^{2}\right)\right]=0.036$

$w R\left(F^{2}\right)=0.098$

$S=1.03$

3111 reflections

182 parameters

0 restraints

Primary atom site location: structure-invariant direct methods
Secondary atom site location: difference Fourier map

Hydrogen site location: difference Fourier map

$\mathrm{H}$-atom parameters constrained

$w=1 /\left[\sigma^{2}\left(F_{\mathrm{o}}^{2}\right)+(0.0527 P)^{2}+0.4861 P\right]$

where $P=\left(F_{\mathrm{o}}^{2}+2 F_{\mathrm{c}}^{2}\right) / 3$

$(\Delta / \sigma)_{\max }=0.001$

$\Delta \rho_{\max }=0.41 \mathrm{e} \AA^{-3}$

$\Delta \rho_{\min }=-0.35$ e $\AA^{-3}$

\section{Special details}

Experimental. ${ }^{1} \mathrm{H}$ NMR $\left(\delta\right.$ p.p.m., $\left.\mathrm{CDCl}_{3}, 400 \mathrm{~Hz}\right)$ : 7.91 (dd, $\mathrm{J}=8.56$ and $\left.2.40 \mathrm{~Hz}, 1 \mathrm{H}\right), 7.47-7.64(\mathrm{~m}, 4 \mathrm{H}), 7.13-7.22(\mathrm{~m}$, $2 \mathrm{H}), 3.11(\mathrm{~s}, 3 \mathrm{H})$.

Geometry. All esds (except the esd in the dihedral angle between two 1.s. planes) are estimated using the full covariance matrix. The cell esds are taken into account individually in the estimation of esds in distances, angles and torsion angles; correlations between esds in cell parameters are only used when they are defined by crystal symmetry. An approximate (isotropic) treatment of cell esds is used for estimating esds involving 1.s. planes.

Refinement. Refinement of $\mathrm{F}^{2}$ against ALL reflections. The weighted R-factor $\mathrm{wR}$ and goodness of fit $\mathrm{S}$ are based on $\mathrm{F}^{2}$, conventional R-factors $R$ are based on $F$, with $F$ set to zero for negative $F^{2}$. The threshold expression of $F^{2}>2 \operatorname{sigma}\left(F^{2}\right)$ is used only for calculating R-factors(gt) etc. and is not relevant to the choice of reflections for refinement. R-factors based on $\mathrm{F}^{2}$ are statistically about twice as large as those based on F, and R- factors based on ALL data will be even larger.

Fractional atomic coordinates and isotropic or equivalent isotropic displacement parameters $\left(\hat{A}^{2}\right)$

\begin{tabular}{|c|c|c|c|c|}
\hline & $x$ & $y$ & $z$ & $U_{\text {iso }} * / U_{\text {eq }}$ \\
\hline $\mathrm{S} 1$ & $0.68157(5)$ & $0.69175(2)$ & $0.61814(4)$ & $0.02500(11)$ \\
\hline $\mathrm{F} 1$ & $1.15704(13)$ & $0.43458(6)$ & $0.91143(11)$ & $0.0407(3)$ \\
\hline $\mathrm{F} 2$ & $0.31417(14)$ & $0.61447(8)$ & $-0.13611(11)$ & $0.0455(3)$ \\
\hline O1 & $0.66160(13)$ & $0.50305(6)$ & $0.36646(11)$ & $0.0228(2)$ \\
\hline $\mathrm{O} 2$ & $0.86399(15)$ & $0.71766(7)$ & $0.69804(14)$ & 0.0380 \\
\hline $\mathrm{C} 1$ & $0.68851(17)$ & $0.59646(8)$ & $0.54180(15)$ & $0.0206(3)$ \\
\hline $\mathrm{C} 2$ & $0.81117(17)$ & $0.53330(8)$ & $0.61313(16)$ & $0.0213(3)$ \\
\hline $\mathrm{C} 3$ & 0.93765 (18) & $0.51943(9)$ & $0.75628(16)$ & $0.0257(3)$ \\
\hline H3 & 0.9568 & 0.5554 & 0.8371 & $0.031 *$ \\
\hline $\mathrm{C} 4$ & 1.03265 (19) & $0.45056(9)$ & $0.77310(17)$ & $0.0284(3)$ \\
\hline $\mathrm{C} 5$ & $1.0128(2)$ & $0.39587(9)$ & 0.65947 (19) & 0.0288 \\
\hline H5 & 1.0839 & 0.3494 & 0.6794 & $0.035 *$ \\
\hline C6 & 0.88870 (19) & $0.40976(8)$ & $0.51726(18)$ & $0.0263(3)$ \\
\hline H6 & 0.8720 & 0.3742 & 0.4364 & $0.032 *$ \\
\hline $\mathrm{C} 7$ & $0.79001(17)$ & $0.47831(8)$ & $0.49901(16)$ & $0.0220(3)$ \\
\hline $\mathrm{C} 8$ & $0.60314(17)$ & $0.57525(8)$ & $0.39537(15)$ & $0.0207(3)$ \\
\hline C9 & $0.46688(17)$ & $0.61298(8)$ & $0.26667(15)$ & 0.0211 \\
\hline $\mathrm{C} 10$ & $0.34606(18)$ & $0.66392(8)$ & $0.28439(16)$ & $0.0231(3)$ \\
\hline $\mathrm{H} 10$ & 0.3529 & 0.6755 & 0.3821 & $0.028 *$ \\
\hline $\mathrm{C} 11$ & 0.21572 (19) & $0.69790(9)$ & $0.16032(17)$ & 0.0263 \\
\hline H11 & 0.1338 & 0.7324 & 0.1737 & $0.032 *$ \\
\hline $\mathrm{C} 12$ & 0.20422 (19) & $0.68181(9)$ & $0.01715(17)$ & $0.0280(3)$ \\
\hline H12 & 0.1159 & 0.7049 & -0.0685 & $0.034 *$ \\
\hline
\end{tabular}


supporting information

\begin{tabular}{lllll}
$\mathrm{C} 13$ & $0.3254(2)$ & $0.63115(10)$ & $0.00347(16)$ & $0.0287(3)$ \\
$\mathrm{C} 14$ & $0.45608(19)$ & $0.59613(9)$ & $0.12251(17)$ & $0.0262(3)$ \\
$\mathrm{H} 14$ & 0.5368 & 0.5615 & 0.1076 & $0.031^{*}$ \\
$\mathrm{C} 15$ & $0.6103(2)$ & $0.66132(11)$ & $0.7597(2)$ & $0.0377(4)$ \\
$\mathrm{H} 15 \mathrm{~A}$ & 0.6903 & 0.6213 & 0.8250 & $0.057^{*}$ \\
$\mathrm{H} 15 \mathrm{~B}$ & 0.4949 & 0.6378 & 0.7123 & $0.057^{*}$ \\
$\mathrm{H} 15 \mathrm{C}$ & 0.6068 & 0.7082 & 0.8193 & $0.057^{*}$ \\
\hline
\end{tabular}

Atomic displacement parameters $\left(\AA^{2}\right)$

\begin{tabular}{lllllll}
\hline & $U^{11}$ & $U^{22}$ & $U^{33}$ & $U^{12}$ & $U^{13}$ & $U^{23}$ \\
\hline S1 & $0.0261(2)$ & $0.01759(17)$ & $0.02614(19)$ & $-0.00056(12)$ & $0.00519(15)$ & $-0.00347(13)$ \\
F1 & $0.0340(5)$ & $0.0452(6)$ & $0.0317(5)$ & $0.0116(4)$ & $0.0017(4)$ & $0.0127(4)$ \\
F2 & $0.0443(6)$ & $0.0705(8)$ & $0.0194(5)$ & $0.0076(5)$ & $0.0105(4)$ & $-0.0019(4)$ \\
O1 & $0.0240(5)$ & $0.0197(5)$ & $0.0223(5)$ & $0.0023(4)$ & $0.0069(4)$ & $-0.0015(4)$ \\
O2 & $0.0311(6)$ & $0.0311(6)$ & $0.0427(7)$ & $-0.0107(5)$ & $0.0055(5)$ & $-0.0085(5)$ \\
C1 & $0.0204(6)$ & $0.0183(6)$ & $0.0215(6)$ & $0.0002(5)$ & $0.0068(5)$ & $0.0003(5)$ \\
C2 & $0.0202(6)$ & $0.0192(6)$ & $0.0240(7)$ & $-0.0003(5)$ & $0.0086(5)$ & $0.0019(5)$ \\
C3 & $0.0245(7)$ & $0.0262(7)$ & $0.0244(7)$ & $-0.0006(6)$ & $0.0077(6)$ & $0.0020(5)$ \\
C4 & $0.0225(7)$ & $0.0312(8)$ & $0.0276(7)$ & $0.0027(6)$ & $0.0061(6)$ & $0.0099(6)$ \\
C5 & $0.0258(7)$ & $0.0230(7)$ & $0.0395(9)$ & $0.0049(5)$ & $0.0152(7)$ & $0.0085(6)$ \\
C6 & $0.0291(7)$ & $0.0198(6)$ & $0.0328(8)$ & $0.0017(5)$ & $0.0153(6)$ & $0.0017(5)$ \\
C7 & $0.0221(6)$ & $0.0197(6)$ & $0.0238(7)$ & $-0.0004(5)$ & $0.0087(6)$ & $0.0021(5)$ \\
C8 & $0.0207(6)$ & $0.0187(6)$ & $0.0229(7)$ & $0.0001(5)$ & $0.0090(5)$ & $-0.0007(5)$ \\
C9 & $0.0188(6)$ & $0.0200(6)$ & $0.0224(7)$ & $-0.0024(5)$ & $0.0061(5)$ & $-0.0002(5)$ \\
C10 & $0.0222(6)$ & $0.0252(7)$ & $0.0214(6)$ & $-0.0003(5)$ & $0.0081(5)$ & $-0.0001(5)$ \\
C11 & $0.0228(7)$ & $0.0245(7)$ & $0.0303(7)$ & $0.0021(5)$ & $0.0092(6)$ & $0.0023(6)$ \\
C12 & $0.0227(7)$ & $0.0314(8)$ & $0.0238(7)$ & $-0.0003(6)$ & $0.0030(6)$ & $0.0062(6)$ \\
C13 & $0.0285(7)$ & $0.0371(8)$ & $0.0190(7)$ & $-0.0035(6)$ & $0.0081(6)$ & $-0.0009(6)$ \\
C14 & $0.0243(7)$ & $0.0301(7)$ & $0.0240(7)$ & $0.0014(5)$ & $0.0095(6)$ & $-0.0021(6)$ \\
C15 & $0.0439(10)$ & $0.0383(9)$ & $0.0378(9)$ & $0.0002(7)$ & $0.0237(8)$ & $-0.0091(7)$ \\
& & & & & &
\end{tabular}

Geometric parameters $\left(\hat{A},{ }^{\circ}\right)$

\begin{tabular}{llll}
\hline $\mathrm{S} 1-\mathrm{O} 2$ & $1.4885(12)$ & $\mathrm{C} 6-\mathrm{C} 7$ & $1.3828(19)$ \\
$\mathrm{S} 1-\mathrm{C} 1$ & $1.7619(14)$ & $\mathrm{C} 6-\mathrm{H} 6$ & 0.9500 \\
$\mathrm{~S} 1-\mathrm{C} 15$ & $1.7880(17)$ & $\mathrm{C} 8-\mathrm{C} 9$ & $1.4614(19)$ \\
$\mathrm{F} 1-\mathrm{C} 4$ & $1.3624(17)$ & $\mathrm{C} 9-\mathrm{C} 10$ & $1.3930(19)$ \\
$\mathrm{F} 2-\mathrm{C} 13$ & $1.3540(17)$ & $\mathrm{C} 9-\mathrm{C} 14$ & $1.399(2)$ \\
$\mathrm{O} 1-\mathrm{C} 8$ & $1.3710(16)$ & $\mathrm{C} 10-\mathrm{C} 11$ & $1.388(2)$ \\
$\mathrm{O} 1-\mathrm{C} 7$ & $1.3754(17)$ & $\mathrm{C} 10-\mathrm{H} 10$ & 0.9500 \\
$\mathrm{C} 1-\mathrm{C} 8$ & $1.3616(19)$ & $\mathrm{C} 11-\mathrm{C} 12$ & $1.385(2)$ \\
$\mathrm{C} 1-\mathrm{C} 2$ & $1.4444(19)$ & $\mathrm{C} 11-\mathrm{H} 11$ & 0.9500 \\
$\mathrm{C} 2-\mathrm{C} 7$ & $1.3949(19)$ & $\mathrm{C} 12-\mathrm{C} 13$ & $1.377(2)$ \\
$\mathrm{C} 2-\mathrm{C} 3$ & $1.395(2)$ & $\mathrm{C} 12-\mathrm{H} 12$ & 0.9500 \\
$\mathrm{C} 3-\mathrm{C} 4$ & $1.372(2)$ & $\mathrm{C} 13-\mathrm{C} 14$ & $1.370(2)$ \\
$\mathrm{C} 3-\mathrm{H} 3$ & 0.9500 & $\mathrm{C} 14-\mathrm{H} 14$ & 0.9500 \\
$\mathrm{C} 4-\mathrm{C} 5$ & $1.390(2)$ & $\mathrm{C} 15-\mathrm{H} 15 \mathrm{~A}$ & 0.9800
\end{tabular}




\begin{tabular}{|c|c|c|c|}
\hline $\mathrm{C} 5-\mathrm{C} 6$ & $1.381(2)$ & $\mathrm{C} 15-\mathrm{H} 15 \mathrm{~B}$ & 0.9800 \\
\hline $\mathrm{C} 5-\mathrm{H} 5$ & 0.9500 & $\mathrm{C} 15-\mathrm{H} 15 \mathrm{C}$ & 0.9800 \\
\hline $\mathrm{O} 2-\mathrm{S} 1-\mathrm{C} 1$ & $106.07(7)$ & $\mathrm{C} 1-\mathrm{C} 8-\mathrm{C} 9$ & $133.75(12)$ \\
\hline $\mathrm{O} 2-\mathrm{S} 1-\mathrm{C} 15$ & $106.34(8)$ & $\mathrm{O} 1-\mathrm{C} 8-\mathrm{C} 9$ & $115.22(11)$ \\
\hline $\mathrm{C} 1-\mathrm{S} 1-\mathrm{C} 15$ & $98.65(7)$ & $\mathrm{C} 10-\mathrm{C} 9-\mathrm{C} 14$ & $119.50(13)$ \\
\hline $\mathrm{C} 8-\mathrm{O} 1-\mathrm{C} 7$ & $106.50(10)$ & $\mathrm{C} 10-\mathrm{C} 9-\mathrm{C} 8$ & $121.44(12)$ \\
\hline $\mathrm{C} 8-\mathrm{C} 1-\mathrm{C} 2$ & $106.99(12)$ & $\mathrm{C} 14-\mathrm{C} 9-\mathrm{C} 8$ & $119.04(12)$ \\
\hline $\mathrm{C} 8-\mathrm{C} 1-\mathrm{S} 1$ & $125.57(11)$ & $\mathrm{C} 11-\mathrm{C} 10-\mathrm{C} 9$ & $120.46(13)$ \\
\hline $\mathrm{C} 2-\mathrm{C} 1-\mathrm{S} 1$ & $126.22(11)$ & $\mathrm{C} 11-\mathrm{C} 10-\mathrm{H} 10$ & 119.8 \\
\hline $\mathrm{C} 7-\mathrm{C} 2-\mathrm{C} 3$ & $119.31(13)$ & $\mathrm{C} 9-\mathrm{C} 10-\mathrm{H} 10$ & 119.8 \\
\hline $\mathrm{C} 7-\mathrm{C} 2-\mathrm{C} 1$ & $104.86(12)$ & $\mathrm{C} 12-\mathrm{C} 11-\mathrm{C} 10$ & $120.49(13)$ \\
\hline $\mathrm{C} 3-\mathrm{C} 2-\mathrm{C} 1$ & $135.77(13)$ & $\mathrm{C} 12-\mathrm{C} 11-\mathrm{H} 11$ & 119.8 \\
\hline $\mathrm{C} 4-\mathrm{C} 3-\mathrm{C} 2$ & $115.84(14)$ & $\mathrm{C} 10-\mathrm{C} 11-\mathrm{H} 11$ & 119.8 \\
\hline $\mathrm{C} 4-\mathrm{C} 3-\mathrm{H} 3$ & 122.1 & $\mathrm{C} 13-\mathrm{C} 12-\mathrm{C} 11$ & $117.62(13)$ \\
\hline $\mathrm{C} 2-\mathrm{C} 3-\mathrm{H} 3$ & 122.1 & $\mathrm{C} 13-\mathrm{C} 12-\mathrm{H} 12$ & 121.2 \\
\hline $\mathrm{F} 1-\mathrm{C} 4-\mathrm{C} 3$ & $117.68(14)$ & $\mathrm{C} 11-\mathrm{C} 12-\mathrm{H} 12$ & 121.2 \\
\hline $\mathrm{F} 1-\mathrm{C} 4-\mathrm{C} 5$ & $117.26(13)$ & $\mathrm{F} 2-\mathrm{C} 13-\mathrm{C} 14$ & $118.00(14)$ \\
\hline $\mathrm{C} 3-\mathrm{C} 4-\mathrm{C} 5$ & $125.05(14)$ & $\mathrm{F} 2-\mathrm{C} 13-\mathrm{C} 12$ & $118.02(14)$ \\
\hline $\mathrm{C} 6-\mathrm{C} 5-\mathrm{C} 4$ & $119.27(13)$ & $\mathrm{C} 14-\mathrm{C} 13-\mathrm{C} 12$ & $123.98(14)$ \\
\hline $\mathrm{C} 6-\mathrm{C} 5-\mathrm{H} 5$ & 120.4 & $\mathrm{C} 13-\mathrm{C} 14-\mathrm{C} 9$ & $117.95(13)$ \\
\hline $\mathrm{C} 4-\mathrm{C} 5-\mathrm{H} 5$ & 120.4 & $\mathrm{C} 13-\mathrm{C} 14-\mathrm{H} 14$ & 121.0 \\
\hline $\mathrm{C} 5-\mathrm{C} 6-\mathrm{C} 7$ & $116.38(14)$ & $\mathrm{C} 9-\mathrm{C} 14-\mathrm{H} 14$ & 121.0 \\
\hline $\mathrm{C} 5-\mathrm{C} 6-\mathrm{H} 6$ & 121.8 & $\mathrm{~S} 1-\mathrm{C} 15-\mathrm{H} 15 \mathrm{~A}$ & 109.5 \\
\hline $\mathrm{C} 7-\mathrm{C} 6-\mathrm{H} 6$ & 121.8 & $\mathrm{~S} 1-\mathrm{C} 15-\mathrm{H} 15 \mathrm{~B}$ & 109.5 \\
\hline $\mathrm{O} 1-\mathrm{C} 7-\mathrm{C} 6$ & $125.23(13)$ & $\mathrm{H} 15 \mathrm{~A}-\mathrm{C} 15-\mathrm{H} 15 \mathrm{~B}$ & 109.5 \\
\hline $\mathrm{O} 1-\mathrm{C} 7-\mathrm{C} 2$ & $110.61(11)$ & $\mathrm{S} 1-\mathrm{C} 15-\mathrm{H} 15 \mathrm{C}$ & 109.5 \\
\hline $\mathrm{C} 6-\mathrm{C} 7-\mathrm{C} 2$ & $124.14(14)$ & $\mathrm{H} 15 \mathrm{~A}-\mathrm{C} 15-\mathrm{H} 15 \mathrm{C}$ & 109.5 \\
\hline $\mathrm{C} 1-\mathrm{C} 8-\mathrm{O} 1$ & $111.03(12)$ & $\mathrm{H} 15 \mathrm{~B}-\mathrm{C} 15-\mathrm{H} 15 \mathrm{C}$ & 109.5 \\
\hline $\mathrm{O} 2-\mathrm{S} 1-\mathrm{C} 1-\mathrm{C} 8$ & $-126.44(13)$ & $\mathrm{C} 1-\mathrm{C} 2-\mathrm{C} 7-\mathrm{C} 6$ & $176.94(13)$ \\
\hline $\mathrm{C} 15-\mathrm{S} 1-\mathrm{C} 1-\mathrm{C} 8$ & $123.68(13)$ & $\mathrm{C} 2-\mathrm{C} 1-\mathrm{C} 8-\mathrm{O} 1$ & $-0.09(15)$ \\
\hline $\mathrm{O} 2-\mathrm{S} 1-\mathrm{C} 1-\mathrm{C} 2$ & $39.24(14)$ & $\mathrm{S} 1-\mathrm{C} 1-\mathrm{C} 8-\mathrm{O} 1$ & $167.87(9)$ \\
\hline $\mathrm{C} 15-\mathrm{S} 1-\mathrm{C} 1-\mathrm{C} 2$ & $-70.64(14)$ & $\mathrm{C} 2-\mathrm{C} 1-\mathrm{C} 8-\mathrm{C} 9$ & $-179.62(14)$ \\
\hline $\mathrm{C} 8-\mathrm{C} 1-\mathrm{C} 2-\mathrm{C} 7$ & $0.90(15)$ & $\mathrm{S} 1-\mathrm{C} 1-\mathrm{C} 8-\mathrm{C} 9$ & $-11.7(2)$ \\
\hline $\mathrm{S} 1-\mathrm{C} 1-\mathrm{C} 2-\mathrm{C} 7$ & $-166.96(10)$ & $\mathrm{C} 7-\mathrm{O} 1-\mathrm{C} 8-\mathrm{C} 1$ & $-0.78(15)$ \\
\hline $\mathrm{C} 8-\mathrm{C} 1-\mathrm{C} 2-\mathrm{C} 3$ & $177.95(15)$ & $\mathrm{C} 7-\mathrm{O} 1-\mathrm{C} 8-\mathrm{C} 9$ & $178.85(11)$ \\
\hline $\mathrm{S} 1-\mathrm{C} 1-\mathrm{C} 2-\mathrm{C} 3$ & $10.1(2)$ & $\mathrm{C} 1-\mathrm{C} 8-\mathrm{C} 9-\mathrm{C} 10$ & $-28.6(2)$ \\
\hline $\mathrm{C} 7-\mathrm{C} 2-\mathrm{C} 3-\mathrm{C} 4$ & $-0.32(19)$ & $\mathrm{O} 1-\mathrm{C} 8-\mathrm{C} 9-\mathrm{C} 10$ & $151.91(12)$ \\
\hline $\mathrm{C} 1-\mathrm{C} 2-\mathrm{C} 3-\mathrm{C} 4$ & $-177.04(15)$ & $\mathrm{C} 1-\mathrm{C} 8-\mathrm{C} 9-\mathrm{C} 14$ & $152.70(16)$ \\
\hline $\mathrm{C} 2-\mathrm{C} 3-\mathrm{C} 4-\mathrm{F} 1$ & $-179.70(12)$ & $\mathrm{O} 1-\mathrm{C} 8-\mathrm{C} 9-\mathrm{C} 14$ & $-26.82(18)$ \\
\hline $\mathrm{C} 2-\mathrm{C} 3-\mathrm{C} 4-\mathrm{C} 5$ & $0.8(2)$ & $\mathrm{C} 14-\mathrm{C} 9-\mathrm{C} 10-\mathrm{C} 11$ & $0.0(2)$ \\
\hline $\mathrm{F} 1-\mathrm{C} 4-\mathrm{C} 5-\mathrm{C} 6$ & $-179.77(13)$ & $\mathrm{C} 8-\mathrm{C} 9-\mathrm{C} 10-\mathrm{C} 11$ & $-178.75(13)$ \\
\hline $\mathrm{C} 3-\mathrm{C} 4-\mathrm{C} 5-\mathrm{C} 6$ & $-0.3(2)$ & $\mathrm{C} 9-\mathrm{C} 10-\mathrm{C} 11-\mathrm{C} 12$ & $-0.2(2)$ \\
\hline $\mathrm{C} 4-\mathrm{C} 5-\mathrm{C} 6-\mathrm{C} 7$ & $-0.7(2)$ & $\mathrm{C} 10-\mathrm{C} 11-\mathrm{C} 12-\mathrm{C} 13$ & $0.3(2)$ \\
\hline $\mathrm{C} 8-\mathrm{O} 1-\mathrm{C} 7-\mathrm{C} 6$ & $-176.95(13)$ & $\mathrm{C} 11-\mathrm{C} 12-\mathrm{C} 13-\mathrm{F} 2$ & $179.38(13)$ \\
\hline $\mathrm{C} 8-\mathrm{O} 1-\mathrm{C} 7-\mathrm{C} 2$ & $1.38(14)$ & $\mathrm{C} 11-\mathrm{C} 12-\mathrm{C} 13-\mathrm{C} 14$ & $-0.2(2)$ \\
\hline $\mathrm{C} 5-\mathrm{C} 6-\mathrm{C} 7-\mathrm{O} 1$ & $179.33(13)$ & $\mathrm{F} 2-\mathrm{C} 13-\mathrm{C} 14-\mathrm{C} 9$ & $-179.58(13)$ \\
\hline
\end{tabular}




\section{supporting information}

$\begin{array}{llll}\mathrm{C} 5-\mathrm{C} 6-\mathrm{C} 7-\mathrm{C} 2 & 1.2(2) & \mathrm{C} 12-\mathrm{C} 13-\mathrm{C} 14-\mathrm{C} 9 & 0.0(2) \\ \mathrm{C} 3-\mathrm{C} 2-\mathrm{C} 7-\mathrm{O} 1 & -179.05(11) & \mathrm{C} 10-\mathrm{C} 9-\mathrm{C} 14-\mathrm{C} 13 & 0.1(2) \\ \mathrm{C} 1-\mathrm{C} 2-\mathrm{C} 7-\mathrm{O} 1 & -1.41(15) & \mathrm{C} 8-\mathrm{C} 9-\mathrm{C} 14-\mathrm{C} 13 & 178.89(13) \\ \mathrm{C} 3-\mathrm{C} 2-\mathrm{C} 7-\mathrm{C} 6 & -0.7(2) & & \end{array}$

Hydrogen-bond geometry $\left(\AA,{ }^{\circ}\right)$

\begin{tabular}{lllll}
\hline$D-\mathrm{H}^{\prime} \cdots A$ & $D-\mathrm{H}$ & $\mathrm{H} \cdots A$ & $D \cdots A$ & $D-\mathrm{H}^{\cdots} A A$ \\
\hline $\mathrm{C} 5-\mathrm{H} 5 \cdots \mathrm{O} 2^{\mathrm{i}}$ & 0.95 & 2.45 & $3.2612(18)$ & 143 \\
$\mathrm{C} 12-\mathrm{H} 12 \cdots 2^{\mathrm{ii}}$ & 0.95 & 2.42 & $3.3361(19)$ & 161 \\
$\mathrm{C} 15-\mathrm{H} 15 A \cdots \mathrm{F}^{\mathrm{iii}}$ & 0.98 & 2.54 & $3.409(2)$ & 147 \\
$\mathrm{C} 15-\mathrm{H} 15 B \cdots \mathrm{F}^{2}$ & 0.98 & 2.55 & $3.163(2)$ & 121 \\
\hline
\end{tabular}

Symmetry codes: (i) $-x+2, y-1 / 2,-z+3 / 2$; (ii) $x-1, y, z-1$; (iii) $-x+2,-y+1,-z+2$; (iv) $x, y, z+1$. 